\title{
La crise de croissance de la plantule de maïs à basse température*
}

\author{
M. Derieux 1, R. Bourdu 2, J.-B. Duburcq ${ }^{1}$ et H. Boizard ${ }^{3}$ \\ 1 INRA, Laboratoire de Génétique et d'Amélioration des Plantes, Estrées-Mons, 80200 Péronne; \\ 2 Université de Paris-Sud, Centre d'Orsay, Structure et Métabolisme des Plantes, 91405 Orsay; \\ ${ }_{3}^{3}$ INRA, Station d'Agronomie Laon-Péronne, Estrées-Mons, 80200 Péronne, France
}

(reçu le 14-10-1987 accepté le 8-11-1988)

Résumé - La crise de croissance étudiée chez la plantule de maïs par Bourdu et Gregory (1983) en conditions de températures favorables se retrouve, accentuée mais au même stade, en conditions froides. Cette crise semble correspondre à l'établissement des translocations vers les racines. Le poids initial et la texture de la semence ont une influence sur la vitesse de croissance et l'utilisation des réserves.

maïs - croissance - stades jeunes - températures basses - qualité de la semence

Summary - Early growth of maize seedlings at low temperatures. The growth crisis of maize seedlings studied by Bourdu and Gregory (1983) under favorable temperatures is accentuated under cold conditions, but at the same stage. This growth crisis seems to be related to the starting of translocation to the roots. The initial weight and the texture of the seeds provide differences in growth rate and in reserves utilization.

maize - early growth - low temperatures - seed quality

\section{Introduction}

La production des maïs précoces est limitée par l'interception du rayonnement lumineux (Bonhomme et al., 1982). Ces maïs, adaptés aux climats du Nord de la France, ont un LAI faible (2 à 2,5 ) aux densités habituelles de culture et cette surface foliaire n'est établie que lorsque le rayonnement solaire est déjà largement décroissant (août) (Varlet-Grancher, 1982). Dans ces conditions, il est particulièrement intéressant d'établir le couvert végétal aussi tôt que possible au printemps (températures basses limitantes) et aussi rapidement que possible.

L'objectif de cette étude est de comparer les résultats obtenus par Bourdu et Gregory (1983) qui ont décrit la période critique de la croissance de la plantule de maïs en conditions favorables $\left(22-25^{\circ} \mathrm{C}\right)$, à ce qui se passe à des températures plus proches de celles que l'on trouve en conditions naturelles au moment du semis $\left(10-15^{\circ} \mathrm{C}\right)$. Cette période est à rapprocher de la période où les agronomes et les sélectionneurs constatent que le maïs "souffre" et utilisent la notion de vigueur au départ (Derieux, 1978).

\section{Matériel et Méthodes}

Trois séries d'expériences (I, II, III) ont été menées en conditions contrôlées à Péronne. Chacune comportait deux traitements : I et III concernaient les semences du même hybride produites sur l'un ou l'autre des parents. L'expérience II concernait des calibres différents du même hybride.

Le choix de l'hybride trois voies HTV 274 pour l'expérience III a été fait pour obtenir, contrairement à l'expérience I, une semence de texture dentée (D), produite sur lignée, plus grosse que la semence cornée (C) du même hybride obtenue sur parent hybride simple. Les principales caractéristiques de ces expériences sont résumées dans le Tableau I. La durée du jour est de $16 \mathrm{~h}$. L'éclairement est de l'ordre de 150 microeinstein par $\mathrm{m}^{2}$ par seconde.

Les semences, pesées individuellement, sont placées dans des godets remplis de vermiculite. L'irrigation est faite par de l'eau jusqu'au $15^{\ominus}$ jour puis par une

\footnotetext{
"Ce travail a bénéficié du soutien de l'AIP INRA «Implantation et croissance juvénile des cultures semées».
} 
Tableau I. Caractéristiques des différentes expériences.

\begin{tabular}{llll}
\hline & \multicolumn{1}{c}{} & II & II \\
\hline Hybride & LG 11 & LG 11 & HTV 274 \\
Parent femelle (semence) & $\mathrm{C}=\mathrm{F}_{7} \times \mathrm{F}_{2}$ & $\mathrm{~F}_{7} \times \mathrm{F}_{2}$ & $\mathrm{C}=\mathrm{F}_{2} \times \mathrm{F}_{251}$ \\
& $\mathrm{D}=\mathrm{W} 401$ & & $\mathrm{D}=\mathrm{F}_{272}$ \\
Poids du grain (en mg) & $\mathrm{C}=299$ & $\mathrm{GC}=350$ & $\mathrm{C}=264$ \\
& $\mathrm{D}=209$ & $\mathrm{PC}=257$ & $\mathrm{D}=330$ \\
Température jour & 14,2 & 15,0 & 15,2 \\
Température nuit & 9,5 & 10,0 & 10,3 \\
\hline
\end{tabular}

$C$ : semences cornées, $D$ : semences dentées, GC : gros calibre, PC : petit calibre.

solution nutritive (solution "spéciale maïs" BB" de Coïc).

Un prélèvement est effectué tous les 3 jours.

Chaque prélèvement correspond à un échantillon de 10 plantes, prises au hasard, prélevées à la même heure, pesées et observées individuellement. Le séchage est effectué à l'étude à $80^{\circ} \mathrm{C}$ pendant $48 \mathrm{~h}$.

\section{Observations}

Parties aériennes: nombre de feuilles visibles et dégainées, poids de matière sèche.

Système racinaire : longueur, nombre, poids de matière sèche pour : racine primaire, racines secondaires, racines latérales et racines coronaires.

Poids de matière sèche du scutellum d'une part, de l'albumen et des téguments d'autre part.

\section{Résultats et discussion}

Les principaux résultats figurent dans le Tableau II.

L'expérience I a été faite dans des conditions un peu plus fraîches $\left(14,2^{\circ} \mathrm{C}\right.$ et $\left.9,5^{\circ} \mathrm{C}\right)$ qui ont entraîné une mortalité assez importante des plantules issues de semences dentées (W401, poids de semence faible). On atteint donc la limite de survie du maïs. Le manque de plantes a entraîné un espacement plus grand des prélèvements tardifs.

Les résultats sont comparables à ceux obtenus à température favorable

Lus 3 séries d'expérience sont très homogènes. On observe en I et III que les semences dentées subissent la crise un peu plus tôt. II ne faut donc comparer, dans un premier temps, que LG 11 sur semences cornées $\left(F_{7} \times F_{2}\right)$, c'est à dire les expériences suivantes : Bourdu et Gregory (1983), IC, II PC et GC,.

La crise de croissance est atteinte dans ces conditions, non plus en 10 jours, mais en 42 à 48 jours (selon la température) et à un stade foliaire comparable : 4 feuilles visibles dont 2 dégainées. L'utilisation des sommes de température comme échelle de temps [par la formule : 0,5 $\left(t_{\max }+\right.$ $\left.t_{\text {Min }}\right)^{-t o]}$ permet d'obtenir les mêmes besoins (280 degrés-jour) pour atteindre la crise en utilisant le seuil de 6. L'homogénéité avec les résultats obtenus à température élevée s'obtient avec un seuil voisin de 9 .

A titre d'exemple, l'évolution du rapport du poids de matière sèche total $\left(P_{\mathrm{x}}\right)$ sur le poids de matière sèche initiale $\left(P_{0}\right)$ est donnée sur la Fig. 1 pour l'expérience IIGC. Ce rapport diminue jusqu'au 30 e jour, jour présumé de démarrage d'une photosynthèse efficace. A ce stade, ce rapport est inférieur à 0,5 , alors qu'à température

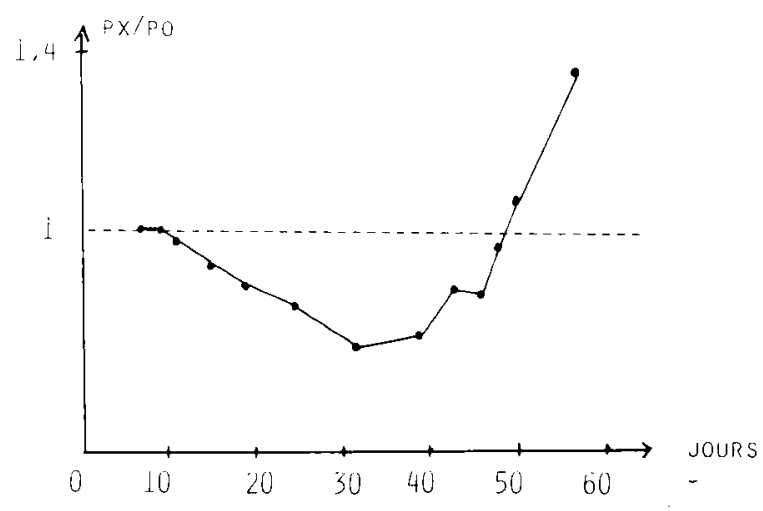

Fig. 1. Evolution du rapport poids de matière sèche totale $\left(P_{\mathrm{x}}\right)$ sur poids de matière sèche initiale $\left(P_{0}\right)$ de la semence pour LG 11, semences cornées GC (expérience II). 
Tableau II. Stade foliaire, nombre de jours, sommes de température au moment de la "crise de croissance" pour les 3 séries d'expérience.

\begin{tabular}{|c|c|c|c|c|c|c|c|}
\hline Expériences & \multicolumn{2}{|l|}{1} & \multicolumn{2}{|l|}{ II } & \multicolumn{2}{|l|}{ III } & $\begin{array}{l}\text { Bourdu et } \\
\text { Gregory (1983) }\end{array}$ \\
\hline Nature de la semence & $\mathrm{C}$ & D & $\mathrm{PC}$ & $\mathrm{GC}$ & $\mathrm{C}$ & $\mathrm{D}$ & c \\
\hline Nombre de jours & 48 & 46 & \multirow{2}{*}{\multicolumn{2}{|c|}{43}} & 42 & 38 & 10 \\
\hline Température & \multicolumn{2}{|c|}{11,85} & & & & & 235 \\
\hline Stades foliaires & 4,4 & 4,0 & 4.1 & 4.0 & \multirow{2}{*}{\multicolumn{2}{|c|}{12,75}} & 4,0 \\
\hline $\begin{array}{l}\text { Feuilles visibles } \\
\text { (dégainées) }\end{array}$ & $(2,0)$ & $(2,0)$ & $(2,1)$ & $(2,0)$ & & & $(2,0)$ \\
\hline \multicolumn{8}{|c|}{ Sommes de degrés $\mathrm{x}$ jour } \\
\hline base 6 & 280 & 269 & 279 & 279 & 283 & 256 & 175 \\
\hline base 9 & 136 & 131 & 150 & 150 & 157 & 142 & 145 \\
\hline
\end{tabular}

élevée, il n'était jamais inférieur à 0,8 . Cela signifie que la plantule consomme davantage de réserves avant que la photosynthèse permette de compenser les pertes respiratoires. Cela est confirmé par la valeur du "résidu", partie de la semence non utilisée pour la croissance de la plantule qui est de $9 \%$ à température basse et de $28 \%$ à température élevée.

La croissance des racines est plus affectée que celle des parties aériennes

Les résultats présentés dans la Fig. 2 montrent de façon très claire que la crise de croissance affecte essentiellement la croissance racinaire. La photosynthèse prend le relais des réserves sans difficulté pour la croissance des feuilles, par contre la croissance des racines est arrêtée entre le $30^{\mathrm{e}}$ jour (jour où globalement la photosynthèse compense les pertes) et le $45^{\mathrm{e}}$ jour. La crise de croissance correspond donc bien à la mise en route de la translocation des produits de la photosynthèse vers les racines (Deleens et al., 1984).

\section{Intérêt d'un "poids de semence» élevé}

Pour l'ensemble des expériences, quels que soient le génotype, la texture et le poids de la semence, l'efficience des réserves est la même (Fig. 3). Pour 60 couples de coefficient de régression est de 0,67 avec $r^{2}=0,99$. Le début de croissance rapide se produit simultanément chez les petites et les grosses semences et pour un même pourcentage d'utilisation des réserves (77\%) (Fig. 4). Cela signifie qu'une quantité plus importante de réserves est utilisée chez les grosses semences et se traduit par une vitesse de croissance plus élevée. La pente de la droite «poids de la plantule en fonction du pourcentage
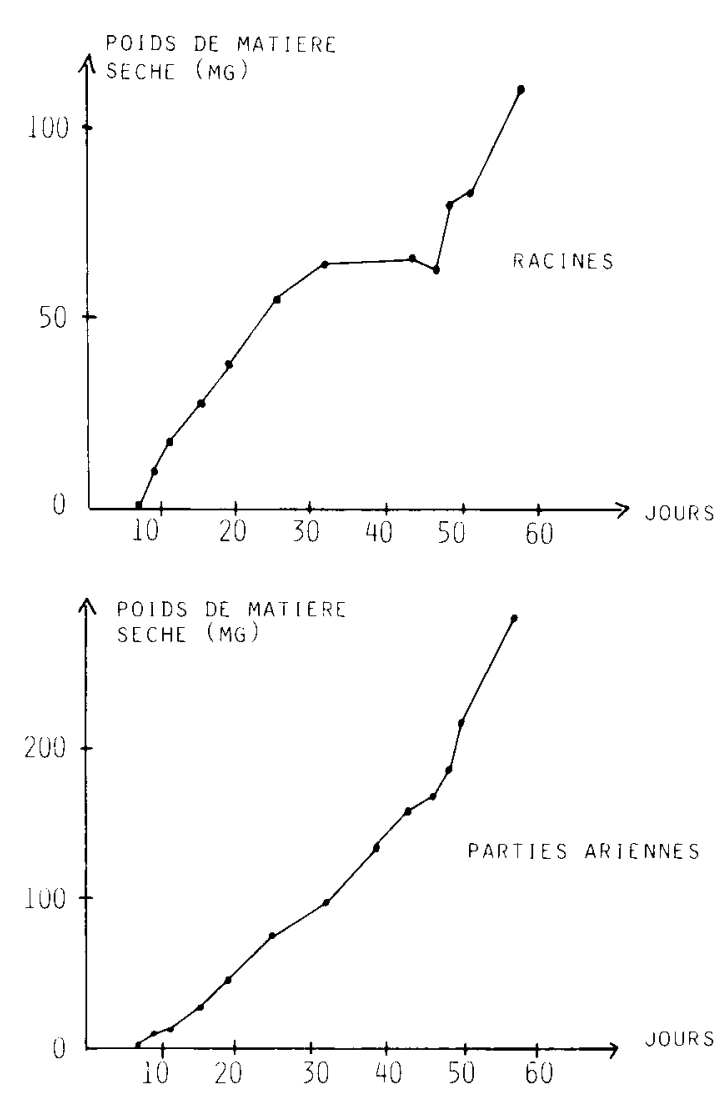

Fig. 2. Croissance comparée partie aérienne, partie souterraine LG 11 , semences cornées GC.

de réserves utilisées" est de 0,41 pour les grosses semences et 0,32 pour les petites semences.

L'avantage lié au poids de la semence signalé par plusieurs auteurs (Chaussat et Le Deunff, 1975; Fok et Chuen 1979; Abd El Rahman et Bourdu, 1986) est ainsi confirmé. 


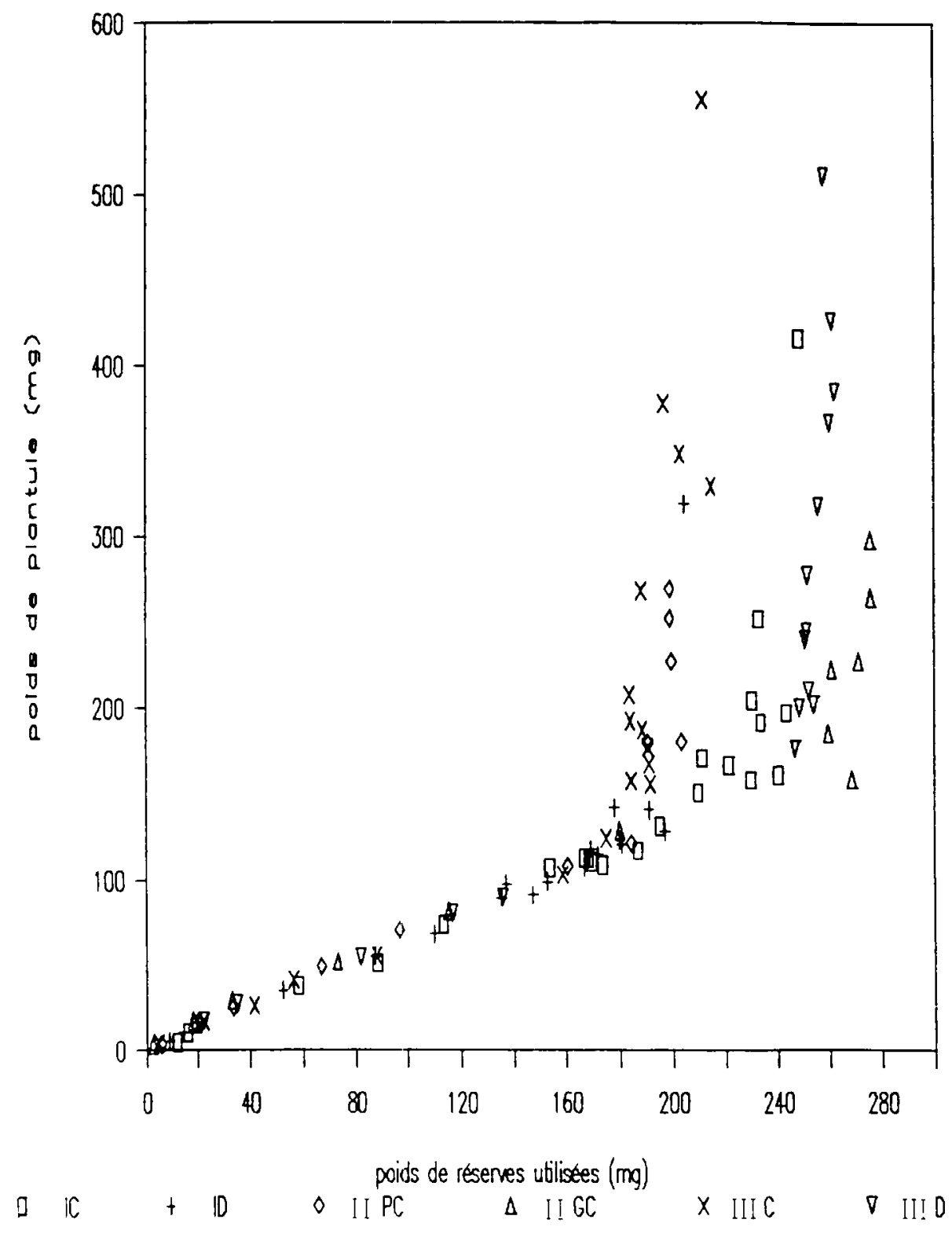

Fig. 3. Poids de plantule en fonction des réserves utilisées.

La taille de la semence ne modifie pas l'allongement de la racine primaire, par contre elle semble modifier l'allongement des racines latérales (Fig. 5).

\section{Influence de la texture de la semence}

Le résidu "réserves + téguments" est légèrement plus élevé pour les semences cornées (Tableau III). L'utilisation des réserves dépend bien de la texture de semence puisque la crojsance rapide (Fig. 6) commence à $75 \%$ des réserves utilisées pour LG 11, semences cornées, et à $90 \%$ des réserves utilisées pour LG 11, semences dentées alors que, pour des semences différant seulement par la taille, ces pourcentages sont les mêmes. La différence de pente est due ici encore à la différence de poids de la semence. Les réserves de semences dentées semblent être consommées plus vite et plus complètement. Cela est intrinsèquement un avantage mais peut être un handicap si les conditions ne permettent pas une photosynthèse efficace après épuisement des réserves.

\section{Conclusion}

La crise de croissance de la plantule de maïs étudiée par Bourdu et Gregory (1983) en conditions de température favorable $\left(25-22^{\circ} \mathrm{C}\right)$ est accentuée en conditions de températures basses $\left(15-10^{\circ} \mathrm{C}\right)$. Dans ces conditions, proches de celles qui existent lors des semis en conditions 
Tableau III. Importance du reliquat en \% des réserves initiales (valeur du reliquat en $\mathrm{mg}$ ).

\section{Expérience I Expérience III}

$\begin{array}{lll}\text { Semences cornées } & 9,0(2,69) & 9,3(2,45) \\ \text { Semences dentées } & 6,0(1,25) & 6,5(2,14)\end{array}$
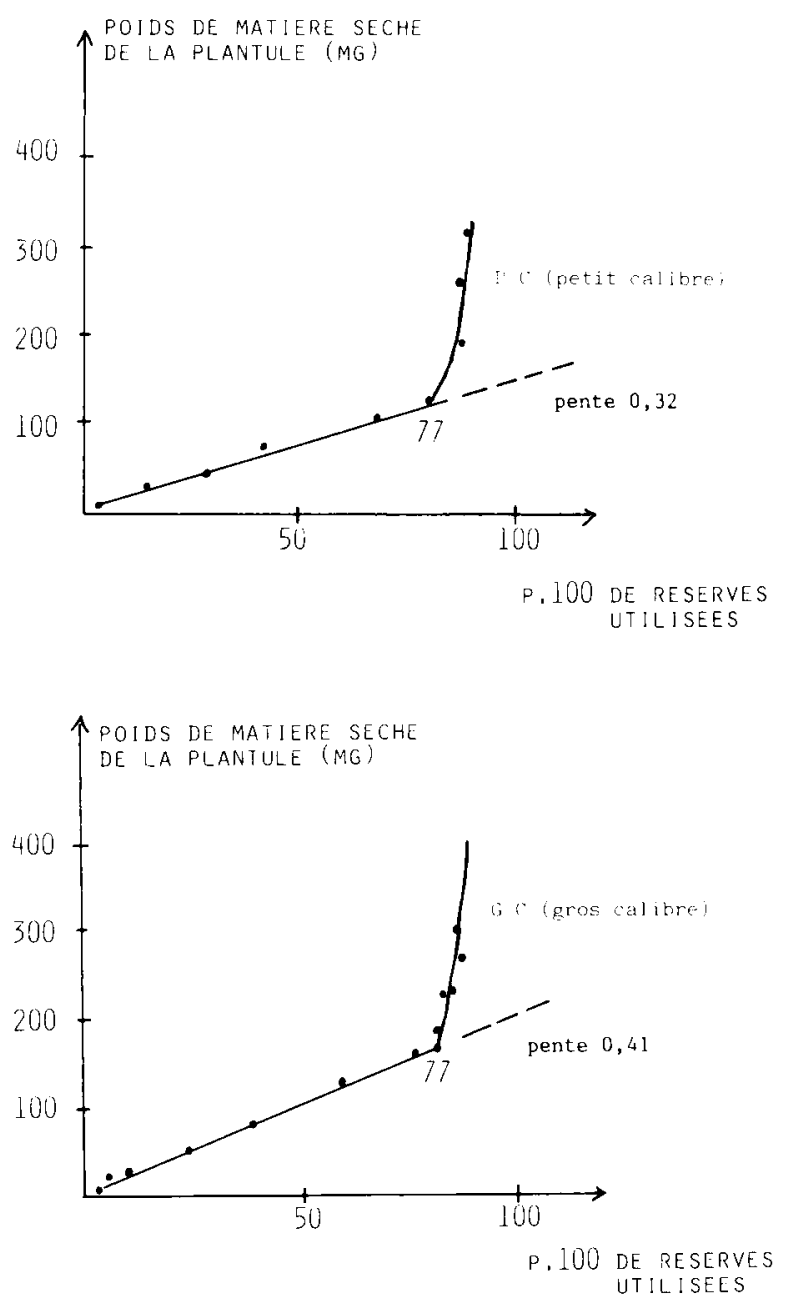

Fig. 4. Influence du poids de la semence (GC, PC) sur la relation entre le pourcentage des réserves utilisées et le poids de la plantule.

naturelles en avril-mai, la quantité (poids de la semence) et/ou la nature des réserves de la semence peuvent modifier les caractéristiques de cette crise de croissance.

Le poids initial de la semence augmente la vitesse de croissance et la taille des organes.

Ce sont surtout les racines qui sont affectées. Cela confirme que cette crise de croissance correspond sans doute à la translocation des produits de la photosynthèse vers les racines.
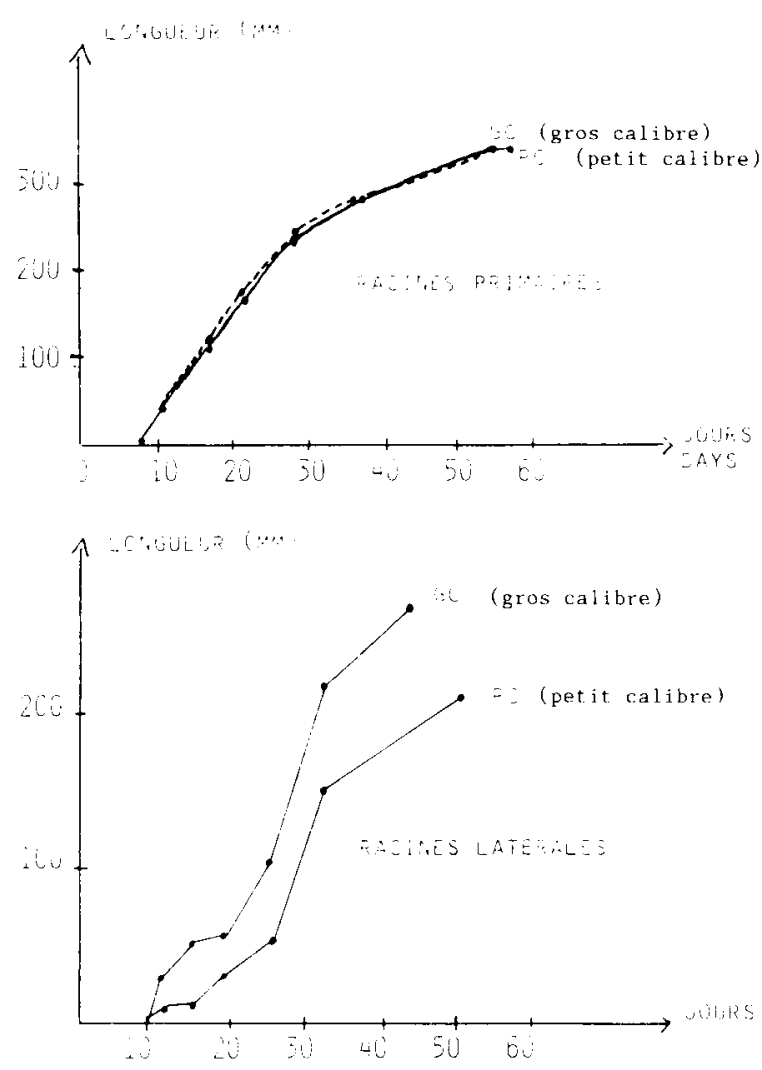

Fig. 5. Effet du poids de la semence (GC, PC) sur l'allongement de la racine primaire et des racines latérales.
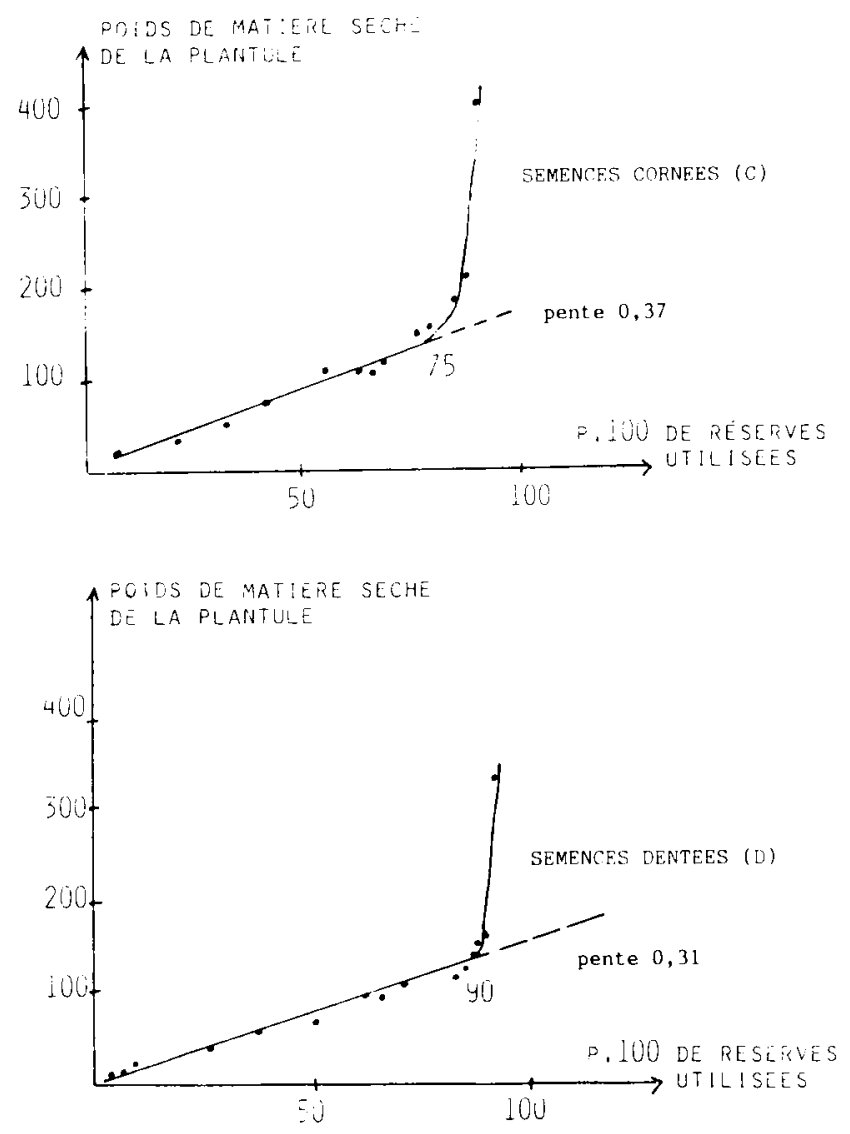

Fig. 6. Influence de la texture sur la croissance de la plantule en fonction du pourcentage de réserves utilisées. 


\section{Références}

Abd El Rahman N. \& Bourdu R. (1986) Effet de la taille et de la forme des grains sur quelques caractéristiques du développement du maïs au stade jeune. Agronomie 6, 181-196

Bonhomme R., Ruget F., Derieux M. \& Vincourt P. (1982) Relations entre production de matière sèche aérienne interceptée chez différents génotypes de maïs. C.R. Acad. Sci. Sér. IIIt. 294, 393-398

Bourdu R. \& Gregory N. (1983) Etude comparée du début de la croissance chez divers génotypes de maïs. Agronomie 3, 761-770

Chaussat R. \& Le Deunff Y. (1975) La prédétermination physiologique des semences. In: La Germination des Semences (Chaussat R. \& Le Deunff T., eds.), Gauthier-Villars, Paris, pp. 219-232

Deleens E., Gregory N. \& Bourdu R. (1984) Transition between seed reserve use and photosynthetic supply during development of maize seedlings. Plant Sci. Lett. 37, 34-39

Derieux M. (1978) Etude de quelques facteurs d'adaptation du maïs aux conditions climatiques du Nord de la France. Ann. Amélior. Plantes 28, 529-566

Fok ah Chuen (1979) Variabilité génétique de la levée et de la vigueur au stade jeune chez le maïs. Mémoire DAA, ENSA, Rennes

Varlet-Grancher C. (1982) Analyse du rendement de la conversion de l'énergie solaire par un couvert végétal. Thèse $n^{\circ} 2593$. Université de Paris-Sud, Orsay 